\title{
Efficiency of Innovative Charge Pump versus Clock Frequency and MOSFETs Sizes
}

\author{
David Matoušek ${ }^{1}$, Jiří Hospodka ${ }^{1}$, Ondřej Šubrt ${ }^{2,1}$ \\ ${ }^{1}$ Department of Circuit Theory, FEE CTU in Prague, Technicka 2, 16627, Prague, Czech Republic, matoudav@fel.cvut.cz \\ ${ }^{2}$ ASICentrum, a company of the Swatch Group, Novodvorska 994, 14221, Prague, Czech Republic
}

\begin{abstract}
Charge pumps are circuits that produce the voltage higher than supply voltage or negative voltage. Today, charge pumps became an integral part of the electronic equipment. The integration of charge pumps directly into the system allows manufacturers to feed a complex system with many specific power requirements from a single source. However, charge pump efficiency is reduced by many phenomena. This paper is focused on the question of efficiency of proposed variant of the charge pump. In this article, the efficiency dependence on a number of stages, output current, clock frequency and MOSFETs sizes was simulated by Eldo. The aim of this study is to determine the MOSFETs sizes and theirs influence to efficiency and the output voltage. Complex optimization of the charge pump circuit will follow in further text.
\end{abstract}

Keywords: 2-phase Charge Pump, non-volatile memory, efficiency, switch size determination.

\section{INTRODUCTION}

The Dickson Charge Pump (DCP) [1] belongs to wellknown charge pump architectures. The design equations for this DCP are summarized in [2]. The differential voltage $\Delta V$ between nodes $n$ and $n+1$ is

$$
\Delta V=V_{n+1}-V_{n}=V_{S}-V_{T}
$$

where $V_{S}$ is the voltage swing at each node due to capacitive coupling from the clock [2], $V_{T}$ is the threshold voltage of the diode-connected transfer transistors.

The optimal value of the voltage swing equals to the amplitude of clocks. But the stray capacitance of node reduces voltage [2] swing as follows

$$
V_{S}=\left(\frac{C_{T}}{C_{T}+C_{S}}\right) \cdot V_{C L K},
$$

where $V_{S}$ is the voltage swing, $C_{T}$ is the transfer capacitance, $C_{S}$ is the stray capacitance, $V_{C L K}$ is the amplitude of clocks.

Since the no-load output voltage applies according to [2]

$$
V_{O}=V_{I N}+N \cdot\left(V_{S}-V_{T}\right)-V_{T}
$$

where $V_{O}$ is the no-load output voltage, $V_{I N}$ is the input voltage, $N$ is the number of stages, $V_{S}$ is the voltage swing, $V_{T}$ is the threshold voltage.
The equation (3) shows the output voltage in an ideal situation when the pump is not delivering any output load current. The effect of the load current is described by [2]

$$
V_{\text {OUT }}=V_{O}-I_{\text {OUT }} \cdot R_{S}
$$

where $V_{\text {OUT }}$ is output voltage at load, $V_{O}$ is the no-load output voltage, $I_{O U T}$ is the load current $\left(I_{O U T} \geq 0\right), R_{S}$ is the internal resistance of the charge pump.

The internal resistance of the charge pump depends on the number of stages $N$, transfer capacitance $C_{T}$, stray capacitance $C_{S}$ and clock frequency $f[2]$

$$
R_{S}=\frac{N}{\left(C_{T}+C_{S}\right) \cdot f},
$$

The threshold voltage of used transistors has usually the main effect to resulted value of the output voltage. It limits the DCP implementation, especially for supply voltage lower than $1 \mathrm{~V}$. Therefore, sub-volt applications use other architectures of charge pumps [3], [4].

A change of connection of transfer transistor from diode mode to switching mode [5], [6], [7] is generally used principle for threshold effect suppression. Thus the voltage drop between two nodes is not a threshold gate-source voltage but the saturation voltage of channel only. 


\section{SUBJECT \& METHODS}

Proposed variant of the charge pump [8] uses the 2-phase clock. One cell as the basic building block of this charge pump is shown in Fig.1. The cell contains five transistors $\left(\mathrm{M}_{1}\right.$ to $\left.\mathrm{M}_{5}\right)$ and transferring capacitor $\left(\mathrm{C}_{\mathrm{T}}\right)$.

This cell is driven by overlapped clock signals according to Fig.2. Falling edges both clock signals start simultaneously.

In the first phase (CLK1 $=V_{\mathrm{DD}}$, CLK2 $\left.=\mathrm{V}_{\mathrm{DD}}\right)$, transistors $\mathrm{M}_{1}, \mathrm{M}_{3}$ and $\mathrm{M}_{5}$ are $\mathrm{ON}$. Thus transferring capacitor $\mathrm{C}_{\mathrm{T}}$ is biased to supply voltage $\mathrm{V}_{\mathrm{DD}}$.

In the second phase (CLK1 $=$ GND, CLK2 $=$ GND), transistors $\mathrm{M}_{2}$ and $\mathrm{M}_{4}$ are $\mathrm{ON}$. Thus transistor $\mathrm{M}_{2}$ holds bias transistor $\mathrm{M}_{5}$ in the disconnected state. Transistor $\mathrm{M}_{4}$ connects the transferring capacitor $\mathrm{C}_{\mathrm{T}}$ between input (output of the previous cell) and output, now. Therefore, the input voltage is increased by a voltage of transferring capacitor from the biased phase.

In the last phase $\left(\mathrm{CLK} 1=\mathrm{GND}, \quad \mathrm{CLK} 2=\mathrm{V}_{\mathrm{DD}}\right)$, all transistors are OFF.

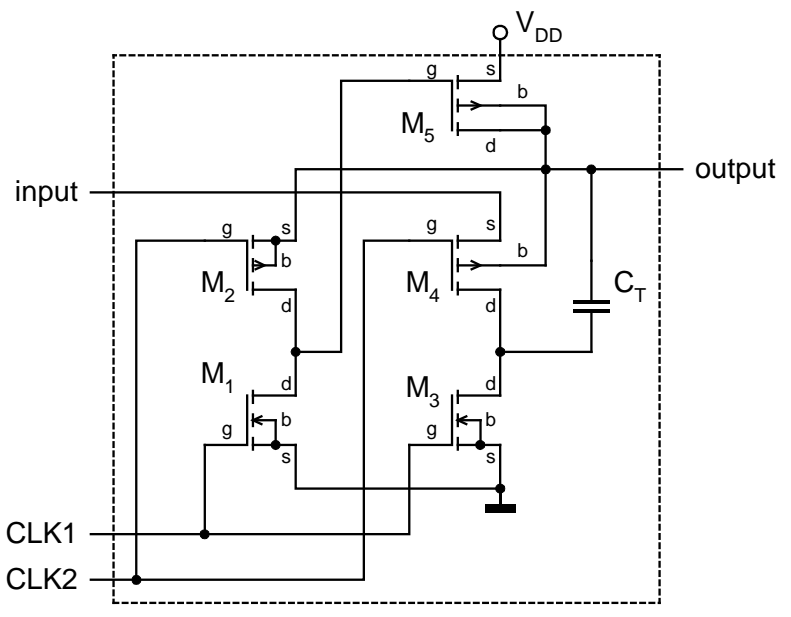

Fig.1. One cell of the proposed charge pump [8].

Used clock signals have overlapped character as is shown in Fig.2. Symbols $W_{1}$ and $W_{2}$ mark width of pulses both clock signals. PER is the period of both clock signals. The optimal $W_{1}, W_{2}$ values for the best ratio between the output voltage and resulted efficiency were estimated in the previous contribution [8] as $W_{1}=24 \mathrm{~ns}$ and $W_{2}=10 \mathrm{~ns}$ for $P E R=50 \mathrm{~ns}$.

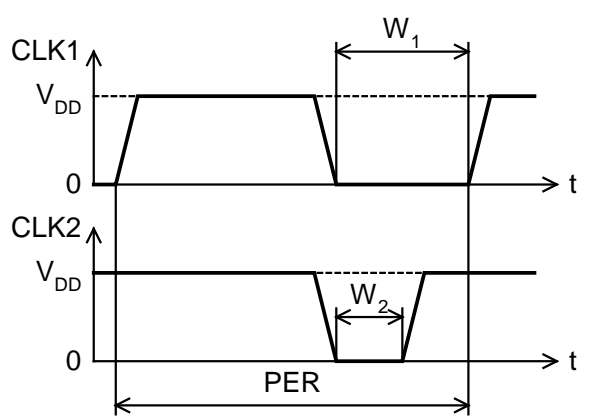

Fig.2. Waveforms of 2-phase clocks.

\section{A. Proposed design algorithm}

The design rules for initial basic parameters estimation of proposed charge pump may be summarized to undermentioned steps.

For illustration, we consider these charge pump specifications:

- power supply voltage $\mathrm{V}_{\mathrm{DD}}=0.7 \mathrm{~V}$, minimal steady-state output voltage $\mathrm{V}_{\text {OUT }}=4 \mathrm{~V}$,

- output load capacitance $\mathrm{C}_{\mathrm{L}}=300 \mathrm{pF}$, output current $\mathrm{I}_{\mathrm{L}}=4 \mu \mathrm{A}$,

- maximal output voltage ramp-up time $t_{r}=150 \mu$ s.

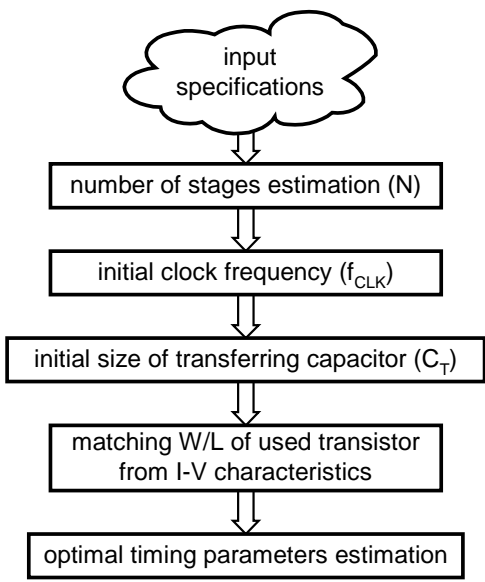

Fig.3. Design-flow diagram.

The number of stages: Initial estimation of number of stages $N$ is calculated as ratio of the output voltage $V_{O U T}$ and the voltage gain of one stage $V_{E}$ (ideally, this gain has the same value as supply voltage):

$$
N=\frac{V_{\text {OUT }}}{V_{E}}=\frac{4}{0.7} \approx 6
$$

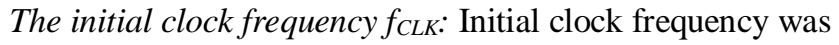
assumed as $f_{C L K}=20 \mathrm{MHz}$.

The initial size of transferring capacitor $C_{T}$ : Value of transfer capacitance $C_{T}$ may be calculated from known load capacitance $C_{L}$, number of stages $N$, ramp-up time $t_{r}$ and clock frequency $f_{C L K}[2]$ :

$$
C_{T}=C_{L} \frac{N}{t_{r} \cdot f_{C L K}}=300 \cdot 10^{-12} \frac{6}{150 \cdot 10^{-6} \cdot 20 \cdot 10^{6}}=0.6 \mathrm{pF}
$$

The W/L of used transistors: The MOSFETs $\mathrm{M}_{3}$ to $\mathrm{M}_{5}$ and $M_{D}$ used for transfer charge should have conductance at least ten times higher than conductance matched to the output current [2], thus their initial size must be estimated from I-V characteristics [8]. The MOSFETs $\mathrm{M}_{1}$ to $\mathrm{M}_{2}$ may cause losses of charge, but these MOSFETs are used for driving transistor $\mathrm{M}_{5}$ only. Therefore, these transistors may be relatively narrow [8].

Parameters of used transistors are summarized in Table 1. 
Transferring capacitor $\mathrm{C}_{\mathrm{T}}$ is realized as transistor $\mathrm{M}_{\mathrm{CT}}$, its capacity was calculated by derivation of $\mathrm{I}-\mathrm{V}$ characteristics. $\mathrm{M}_{\mathrm{BUFA}}$ and $\mathrm{M}_{\mathrm{BUFB}}$ are transistors from clock buffers. Models nmos_hvt and pmos_hvt correspond to "high voltage" transistors with a relatively high value of $\mathrm{V}_{\mathrm{T}}$. Model nmos_na18v corresponds to the native transistor for $1.8 \mathrm{~V}$ technology.

Table 1. Parameters of transistors.

\begin{tabular}{|c|c|c|c|}
\hline Transistor & $\mathbf{W}(\boldsymbol{\mu m})$ & $\mathbf{L}(\boldsymbol{\mu m})$ & model \\
\hline $\mathrm{M}_{1}$ & 0.2 & 0.1 & nmos_hvt \\
\hline $\mathrm{M}_{2}$ & 1 & 0.1 & pmos_hvt \\
\hline $\mathrm{M}_{3}$ & 0.5 & 0.1 & nmos_hvt \\
\hline $\mathrm{M}_{4}$ & 2.5 & 0.1 & pmos_hvt \\
\hline $\mathrm{M}_{5}$ & 2.5 & 0.1 & pmos_hvt \\
\hline $\mathrm{M}_{\mathrm{CT}}$ & 30 & 10 & nmos_hvt \\
\hline $\mathrm{M}_{\mathrm{D}}$ & 20 & 0.8 & nmos_na18v \\
\hline $\mathrm{M}_{\text {BUFA }}$ & 5 & 0.1 & nmos_hvt \\
\hline $\mathrm{M}_{\text {BUFB }}$ & 12.5 & 0.1 & pmos_hvt \\
\hline
\end{tabular}

The estimated area of the chip for the realization of proposed charge pump is listed in Table 2. where $N$ is the number of stages, $A_{S T A G E S}$ is the area for the realization of stages, $A_{B U F D E T}$ is the area for realization clock buffers and output detector, $A_{\text {TотаL }}$ is the area for realization proposed charge pump with required number of stages. The technological node corresponds to $100 \mathrm{~nm}$.

Table 2. Estimated area for the realization of the charge pump.

\begin{tabular}{|c|c|c|c|}
\hline $\mathbf{N}$ & Astages $\left(\boldsymbol{\mu \mathbf { m } ^ { 2 } )}\right.$ & A bufdet $\left(\boldsymbol{\mu \mathbf { m } ^ { 2 } )}\right.$ & A $_{\text {total }}\left(\boldsymbol{\mu \mathbf { m } ^ { 2 }}\right)$ \\
\hline 1 & 469 & 30 & 499 \\
\hline 2 & 998 & 30 & 1028 \\
\hline 3 & 1497 & 30 & 1527 \\
\hline 4 & 1996 & 30 & 2026 \\
\hline 5 & 2495 & 30 & 2525 \\
\hline 6 & 2994 & 30 & 3024 \\
\hline
\end{tabular}

Note, that area for realization clock buffers and output detector $A_{B U F D E T}$ has a constant value over a number of stages $N$. Namely, individual stages of the charge pump use common clock buffers.

The optimal timing parameters: The optimal timing parameters for clock frequency $f_{C L K}=20 \mathrm{MHz}$ were estimated in previous contribution [8] as $W_{1}=24 n s$ and $W_{2}=10 \mathrm{~ns}$ for $P E R=50 \mathrm{~ns}$. For other values of frequency, the timing parameters must be proportionally changed according to Table 3.

Table 3. Timing parameters for various value of clock frequency.

\begin{tabular}{|c|c|c|c|}
\hline $\mathbf{f}_{\text {CLK }}$ (MHz) & PER (ns) & $\mathbf{W}_{\mathbf{1}}(\mathbf{n s )}$ & $\mathbf{W}_{\mathbf{2}}$ (ns) \\
\hline 10 & 100 & 48 & 20 \\
\hline 13.3 & 75 & 36 & 15 \\
\hline 20 & 50 & 24 & 10 \\
\hline 27 & 37 & 17.8 & 7.4 \\
\hline 40 & 25 & 12 & 5 \\
\hline
\end{tabular}

B. Study of clock frequency influence to efficiency and output voltage

The aim of this part of simulations is to determine the influence of clock frequency to efficiency primarily and output voltage secondarily.

Schematic diagram of simulated circuit is shown in Fig.4. This instance is 6-stage charge pump $(\mathrm{N}=6)$. A number of stages (i.e. cells) are changed for other instances only. Clock signals are buffered by strong buffers (inverters). Diode detector based on transistor $M_{D}$ is connected to the last stage. Resistor $\mathrm{R}_{\mathrm{L}}$ and capacitor $\mathrm{C}_{\mathrm{L}}$ model resistive and capacitive parts of output load. Proposed charge pump was powered from $V_{D D}=0.7 \mathrm{~V}$ and both clock signals had amplitude $0.7 \mathrm{~V}$, too. Symbol $I_{S}$ marks consumed current. The output voltage at the load is marked $V_{\text {OUT }}$.

A number of stages $\mathrm{N}$ were varied from 1 to 6 , clock frequency was set to values 10,13.3, 20, 27, $40 \mathrm{MHz}$. Complex analyse for a number of stages varied from 1 to 6 was performed. But in this text, the results for variant $\mathrm{N}=6$ was chosen. Symbol $\mathrm{I}_{\mathrm{L}}$ marks resistive part of output current.

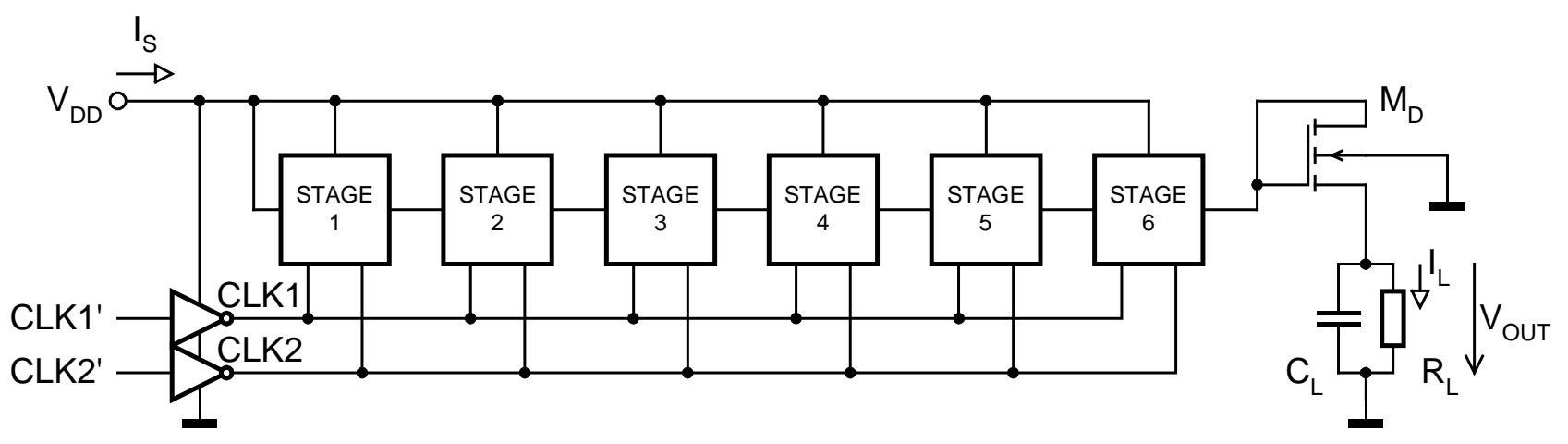

Fig.4. Simplified schematic diagram of the simulated charge pump for N=6. 
Fig.5. shows that efficiency increases with increasing value of load current and decreasing value of clock frequency. Increasing the clock frequency leads to increasing power consumption, thus efficiency decreases with increasing clock frequency. While the power consumption for given clock frequency is relatively near a constant value and does not vary with output current. Therefore, efficiency increases with increasing load current.

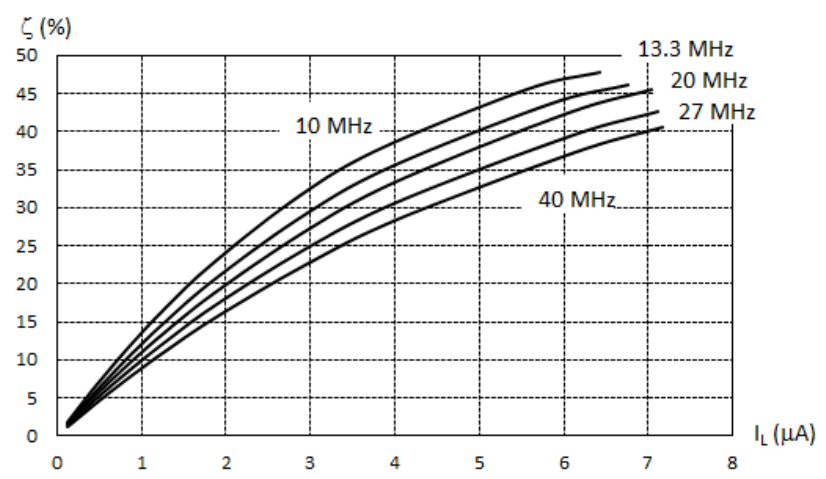

Fig.5. Efficiency as a function of $I_{L}$ for $\mathrm{N}=6$.

Fig.6. shows the output voltage variation with resistive part of output current $\mathrm{I}_{\mathrm{L}}$ and clock frequency. Increasing the clock frequency will increase the amount of charge transferred over a given time interval, thus output voltage has a higher value. The presence of a fixed capacitive load leads to increasing no-load output voltage with increasing clock frequency.

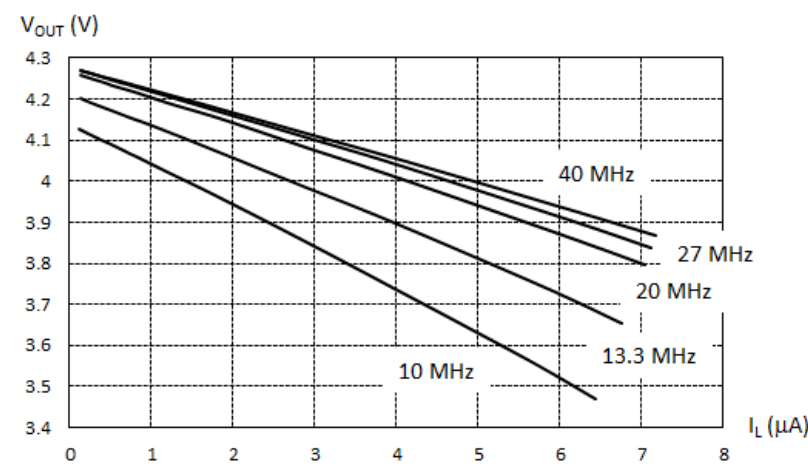

Fig.6. Output voltage as a function of $I_{L}$ for $\mathrm{N}=6$.

Total efficiency was calculated as ratio of the output power $P_{\text {OUT }}$ in steady-state (8) and the total consummated power from power supply $P_{I N}(9)$ according to (10)

$$
\begin{gathered}
P_{\text {OUT }}=V_{\text {OUT }} \cdot I_{\text {OUT }}=\frac{V_{\text {OUT }}^{2}}{R_{L}}, \\
P_{I N}=V_{D D} \cdot I_{S}, \\
\zeta=\frac{P_{I N}}{P_{\text {OUT }}} \cdot 100 \%=\frac{V_{\text {OUT }}^{2}}{V_{D D} \cdot I_{S} \cdot R_{L}} \cdot 100 \%,
\end{gathered}
$$

where $V_{O U T}$ is the output voltage in steady-state, $V_{D D}$ is the power voltage, $I_{S}$ is the average value of current consumed from power supply including a current of clock buffers, $R_{L}$ is the output load.

Efficiency is varied about $10 \%$ (from $38.47 \%$ at frequency $10 \mathrm{MHz}$ to $28.64 \%$ at frequency $40 \mathrm{MHz}$ ) for the nominal value of the output current $I_{L}=4 \mu \mathrm{A}$. For the same conditions, the output voltage varies from $3.741 \mathrm{~V}$ to $4.051 \mathrm{~V}$.

Simulation results are very similar for another number of stages of the charge pump. The situation is summarized in Table 4.

Table 4. Efficiency and output voltage for a various number of stages at output current $4 \mu \mathrm{A}$.

\begin{tabular}{|c|c|c|}
\hline $\mathbf{N}$ & $\boldsymbol{\zeta ( \% )}$ & Vout $(\mathbf{V})$ \\
\hline 1 & 69.53 to 59.33 & 1.275 to 1.290 \\
\hline 2 & 64.63 to 43.26 & 1.919 to 1.950 \\
\hline 3 & 60.06 to 47.07 & 2.540 to 2.590 \\
\hline 4 & 53.75 to 42.02 & 3.130 to 3.120 \\
\hline 5 & 47.79 to 37.30 & 3.638 to 3.762 \\
\hline 6 & 37.47 to 28.64 & 3.671 to 4.051 \\
\hline
\end{tabular}

Values of efficiency are relatively low because proposed charge pump works at relatively low value of the power supply voltage $\left(\mathrm{V}_{\mathrm{DD}}=0.7 \mathrm{~V}\right)$.

\section{MOSFET size influence to efficiency and output voltage}

The aim of this part of simulations is to determine the influence of MOSFETs size to the efficiency and output voltage. Thus sizes of all transistors were swept in the second part of simulations. Only one parameter was changed, sizes of other transistors (according to Table 1.) were left unchanged. Output load was set to constant value $\mathrm{R}_{\mathrm{L}}=1 \mathrm{M} \Omega$. These simulations were performed for 6-stage charge pump only at clock frequency $20 \mathrm{MHz}$.

Next, the seven options of simulations were performed to determine the influence of transistor dimensions.

Option 1: width of transistors $\mathrm{M}_{4}$ and $\mathrm{M}_{5}$ was swept from $0.5 \mu \mathrm{m}$ to $50 \mu \mathrm{m}$. The size of these transistors has strong influence to both observed quantities (see Fig.7.) because these transistors have the main effect to transfer of charge in the first phase (biasing of $\mathrm{C}_{\mathrm{T}}$ ) and the second phase (transferring charge of $\mathrm{C}_{\mathrm{T}}$ to output).

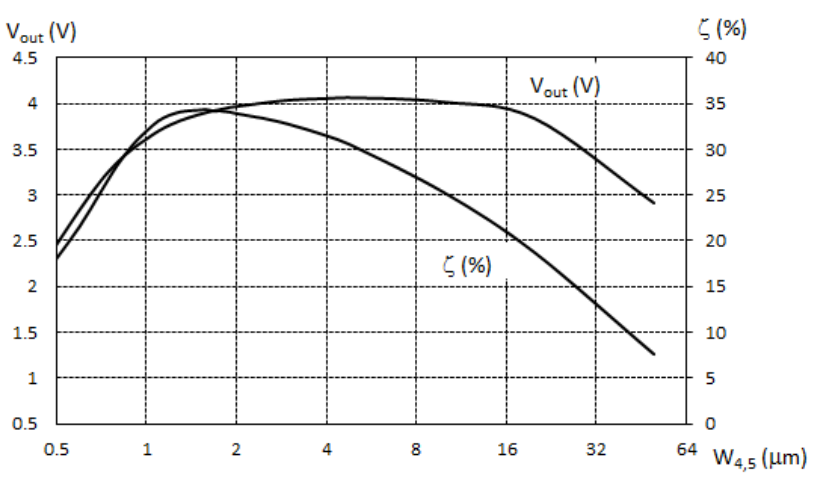

Fig.7. Option 1: Efficiency and output voltage as functions of $W_{4,5}$. 
Option 2: width of detector $\mathrm{M}_{\mathrm{D}}$ was swept from $0.5 \mu \mathrm{m}$ to $50 \mu \mathrm{m}$. The size of the transistor $M_{D}$ has the primarily influence to the efficiency (see Fig.8.) because this transistor determines the ratio of energy that is transmitted to load to the energy that is consumed from the power supply.

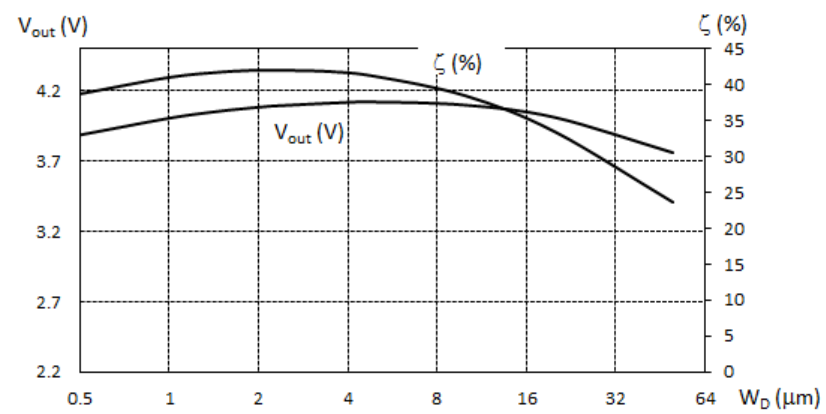

Fig.8. Option 2: Efficiency and output voltage as functions of $W_{D}$.

Option 3: width of transistor $\mathrm{M}_{3}$ was swept from $0.2 \mu \mathrm{m}$ to $10 \mu \mathrm{m}$. The size of the transistor $\mathrm{M}_{3}$ has the primarily influence to the output voltage (see Fig.9.), because this transistor has significant effect to transfer of charge in the first phase (biasing of $\mathrm{C}_{\mathrm{T}}$ ).

Option 4, 5: widths of transistors $\mathrm{M}_{1}$ and $\mathrm{M}_{2}$ were swept from $0.2 \mu \mathrm{m}$ to $10 \mu \mathrm{m}$ or from $0.2 \mu \mathrm{m}$ to $20 \mu \mathrm{m}$ respectively. Increasing widths of these transistors lead to an increase of discharging process effect of the transfer capacitor $\mathrm{C}_{\mathrm{T}}$. Hence, transistors $\mathrm{M}_{1}$ and $\mathrm{M}_{2}$ must be narrowed, see Fig. 10 .

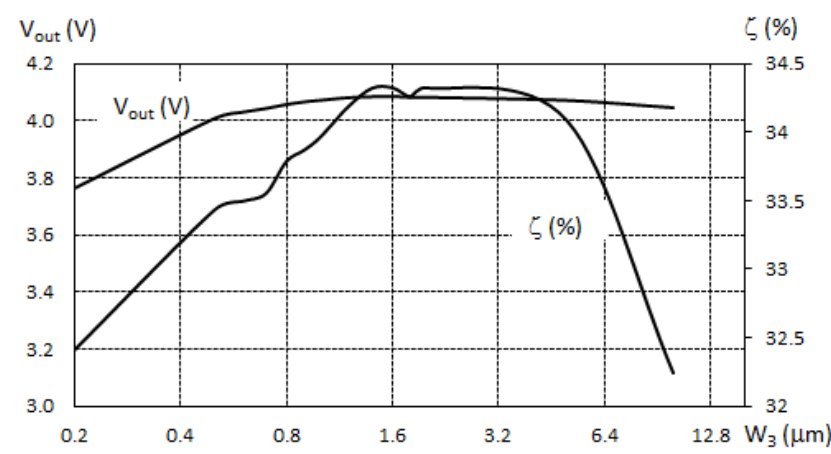

Fig.9. Option 3: Efficiency and output voltage as functions of $W_{3}$.

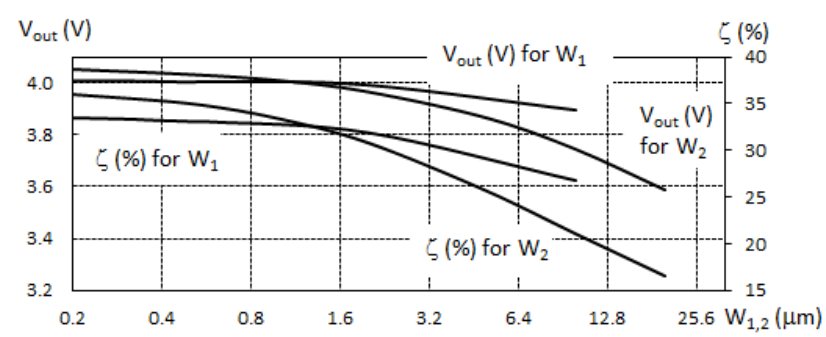

Fig.10. Option 4, 5: Efficiency and output voltage as functions of $W_{1,2}$.
Option 6: width of transistor $\mathrm{M}_{\mathrm{CT}}$ was swept from $6 \mu \mathrm{m}$ to $60 \mu \mathrm{m}$. The optimal size of the transistor $\mathrm{M}_{\mathrm{CT}}$ is a compromise between efficiency and output voltage (see Fig.11.). Influence of variation in the capacity of transfer capacitor $C_{T}$ has a strong effect on the internal resistance of the charge pump (thus this capacity has a strong effect on the output voltage). The optimal value of $\mathrm{C}_{\mathrm{T}}$ capacity for maximizing efficiency is a more complex question. The main role plays value of $\mathrm{C}_{\mathrm{S}}$ capacity for low values of $\mathrm{C}_{\mathrm{T}}$ and increased power supply for high values of $\mathrm{C}_{\mathrm{T}}$.

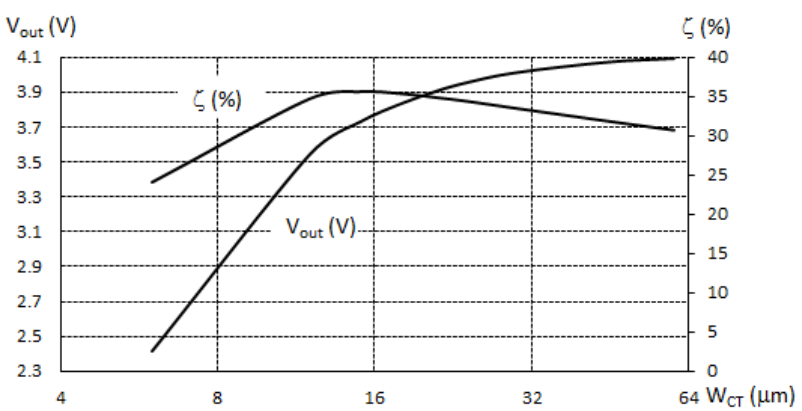

Fig.11. Option 6: Efficiency and output voltage as functions of $W_{C T}$.

Option 7: width of transistor $\mathrm{M}_{\mathrm{BUFA}}$ was swept from $1 \mu \mathrm{m}$ to $20 \mu \mathrm{m}$ and simultaneously $\mathrm{M}_{\mathrm{BUFB}}$ was swept from $5 \mu \mathrm{m}$ to $100 \mu \mathrm{m}$. Maximal value of efficiency $\zeta=33.67 \%$ for $V_{\text {OUT }}=4.006 \mathrm{~V}$ occurs for $W_{B U F A}=1 \mu \mathrm{m}$ and $W_{B U F B}=5 \mu \mathrm{m}$. This variant isn't plotted because differences of values of efficiency and output voltage were relatively small. These transistors must be narrowed because increased conductance of wide transistor leads to increase switching current of these buffers.

\section{RESULTS}

Results from the simulations imply possibility for optimization efficiency of proposed charge pump. Values of efficiency and output voltage are $\zeta=33.43 \%$ and $V_{O U T}=4.008 \mathrm{~V}$ for original sizes of MOSFETs according to Table 1. and load $\mathrm{R}_{\mathrm{L}}=1 \mathrm{M} \Omega$.

\section{DisCUSSION}

Study of efficiency and the output voltage versus clock frequency and MOSFETs sizing for proposed charge pump was performed. Parameters of MOSFETs and clock scheme were chosen according to a previous study [8]. Analyse was performed by Eldo simulator version 2010.2b. The Eldo is an SPICE-like simulator from Mentor Graphics Corporation.

The first part of simulations demonstrates that the efficiency increases with increasing load current and decreasing clock frequency. However, the dependency of the output voltage has opposite character, because the output voltage increases with decreasing load current and increasing clock frequency. 
Results from the second part of simulations imply possibility for optimization efficiency of proposed charge pump. Effect of transistor sizing allows improving the efficiency about ten percent. Dimensions of transistors $M_{4}$, $M_{5}, M_{D}$ and $M_{C T}$ have the main effect on efficiency, $M_{3}$ has the important effect on the output voltage. Effect of other transistors has predictable character and we may neglect effect on the size of these transistors. Results from this study can be used for complex optimization study performed in the next period.

The validity of the presented results is limited to pre-layout simulations. Therefore, parasitic effects of higher orders (e.g. interlayer capacitance, metallic interconnection capacitance etc.) are neglected. These parasitic effects can have a significant influence on attainable results.

\section{ACKNOWLEDGMENT}

This work has been supported by the grant No. SGS14/191/OHK3/3T/13 of the CTU in Prague.

\section{REFERENCES}

[1] Dickson, J.F. (1976). On-Chip high-voltage generation in NMOS integrated circuits using an improved voltage multiplier technique. IEEE Journal of SolidState Circuits, 11 (3), 374-378.

[2] Pan, F., Samaddar, T. (2006). Charge Pump Circuit Design. McGraw-Hill Education.

[3] Palumbo, G., Pappalardo, D. (2010). Charge pump circuits: An overview on design strategies and topologies. IEEE Circuits and Systems Magazine, 10 (1), 31-45.

[4] Matousek, D. (2014). Comparison of selected architectures of negative Charge Pumps with new design. In Radioelektronika : 24th International Conference, April 15-16, 2014. IEEE.

[5] Yamazoe, T., Ishida, H., Nihongi, Y. (2009). A Charge Pump that generates positive and negative high voltages with low power-supply voltage and low power consumption for non-volatile memories. In International Symposium on Circuits and Systems (ISCAS 2009), May 24-27, 2009. IEEE, 988-999.

[6] Wong, O.Y., Wong, R., Tam, W.S., Kok, C.W. (2011). An overview of charge pumping circuits for Flash memory applications. In 9th International Conference on ASIC (ASICON), October 25-28, 2011. IEEE, 116119.

[7] Wong, O.Y., Wong, H., Tam, W.S., Kok, C.W. (2014). On the design of power- and area-efficient Dickson charge pump circuits. Analog Integrated Circuits and Signal Processing, 78 (2), 373-389.

[8] Matousek, D., Subrt, O., Hospodka, J. (2015). Charge pump design for use in NVM device test and measurement. In MEASUREMENT 2015: 10th International Conference, May 25-28, 2015. Institute of Measurement Science SAS, 203-206.

Received March 29, 2016. Accepted October 07, 2016. 